\title{
ON THE ORIGIN OF THE SALPETER SLOPE FOR THE INITIAL MASS FUNCTION
}

\author{
M. S. OEY \\ Department of Astronomy, University of Michigan, 830 Dennison Building, 500 Church Street, Ann Arbor, MI 48109-1042, USA \\ Received 2011 June 15; accepted 2011 August 4; published 2011 September 7
}

\begin{abstract}
We suggest that the intrinsic, stellar initial mass function (IMF) follows a power-law slope $\gamma=2$, inherited from hierarchical fragmentation of molecular clouds into clumps and clumps into stars. The well-known, logarithmic Salpeter slope $\Gamma=1.35$ in clusters is then the aggregate slope for all the star-forming clumps contributing to an individual cluster, and it is steeper than the intrinsic slope within individual clumps because the smallest star-forming clumps contributing to any given cluster are unable to form the highest-mass stars. Our Monte Carlo simulations demonstrate that the Salpeter power-law index is the limiting value obtained for the cluster IMF when the lower-mass limits for allowed stellar masses and star-forming clumps are effectively equal, $m_{\mathrm{lo}}=M_{\mathrm{lo}}$. This condition indeed is imposed for the high-mass IMF tail by the turnover at the characteristic value $m_{c} \sim 1 M_{\odot}$. IMF slopes of $\Gamma \sim 2$ are obtained if the stellar and clump upper-mass limits are also equal $m_{\text {up }}=M_{\text {up }} \sim 100 M_{\odot}$, and so our model explains the observed range of IMF slopes between $\Gamma \sim 1$ and 2 . Flatter slopes of $\Gamma=1$ are expected when $M_{\mathrm{lo}}>m_{\mathrm{up}}$, which is a plausible condition in starbursts, where such slopes are suggested to occur. While this model is a simplistic parameterization of the star formation process, it seems likely to capture the essential elements that generate the Salpeter tail of the IMF for massive stars. These principles also likely explain the integrated galaxy IMF (IGIMF) effect seen in low-density star-forming environments.
\end{abstract}

Key words: galaxies: star clusters: general - galaxies: stellar content - open clusters and associations: general stars: formation - stars: luminosity function, mass function - stars: massive - stars: statistics

\section{INTRODUCTION}

The mass distribution of stars at birth, known as the initial mass function (IMF), is perhaps the most important fundamental parameterization of the star formation process (e.g., Lada 2009; McKee \& Ostriker 2007; Adams 2003). When Salpeter (1955) first empirically evaluated the IMF, he identified a simple powerlaw distribution for stellar mass $m$, having exponent $\gamma=2.35$ :

$$
n(m) d m \propto m^{-\gamma} d m .
$$

While it is now clear that the IMF has a characteristic mass below which its form turns over, yielding additional distinct regimes at lower masses (e.g., Kroupa 2001; Chabrier 2003), Salpeter's original prescription nevertheless has remained surprisingly robust for $m \gtrsim 1 M_{\odot}$. Since the stellar light from galaxies is dominated by these more luminous stars, the "Salpeter IMF" has proven vital to the entire field of galaxy evolution, in addition to star formation and stellar populations.

Yet, more than half a century after Salpeter's (1955) seminal work, the physical factors yielding the value of $\gamma=2.35$ for the power-law index remain elusive and poorly understood. The origin of the IMF continues to be a topic of intense discussion, and the reader is referred to recent reviews by Lada (2009), Clarke (2009), Elmegreen (2009), McKee \& Ostriker (2007), and Bonnell et al. (2007) for comprehensive overviews on IMF theory.

One obstacle to gaining deeper physical understanding is the fact that the Salpeter slope emerges from a variety of simulations dominated by different mechanisms. In particular, both the core collapse (e.g., Krumholz et al. 2010) and competitive accretion scenarios (e.g., Bonnell et al. 2003) are able to reproduce the Salpeter slope. Effects that are explored in modern simulations include decaying versus driven turbulence, isothermal versus non-isothermal equations of state, and inclusion or exclusion of magnetic fields, among other factors; the IMF slope generally does not provide a strong discriminant on this large parameter space (e.g., Clarke 2009; McKee \& Ostriker 2007).

Here, we suggest an explanation of the Salpeter slope that may provide the simplest basic physical understanding, but which may correspond to a framework within which the more specific physics of actual star formation operates.

\section{FROM THE CLUSTER MASS FUNCTION TO THE IMF}

Most, if not all, stars form in clusters (e.g., McKee \& Ostriker 2007; Lada \& Lada 2003), which in turn form with an initial cluster mass function (ICMF). Like the stellar IMF, the cluster ICMF is also described well by a power-law distribution in cluster mass $M$ :

$$
N(M) d M \propto M^{-\beta} d M .
$$

Again like the stellar IMF, the ICMF power-law index is also found to be robust and largely invariant, but slightly shallower, $\beta \sim 2.0$, than the Salpeter slope, based on clusters covering a wide range of scales, including super star clusters (e.g., Meurer et al. 1995), massive and open clusters (e.g., Elmegreen \& Efremov 1997; Zhang \& Fall 1999; de Grijs et al. 2003), sparse OB groups (Oey et al. 2004), and the H II region luminosity function (e.g., Oey \& Clarke 1998; Kennicutt et al. 1989).

The similarity in the slopes of the IMF and ICMF, $\gamma=2.35$ versus $\beta=2.0$, respectively, has been previously noted (e.g., Elmegreen 2006). Since star formation is a hierarchical process, with smaller units fragmenting from larger ones, we can examine the origin of the IMF as the relationship between the IMF and ICMF. The ICMF slope $\beta=2$ is a value that seems simpler to understand. As is often pointed out, this power-law index corresponds to a uniform distribution of power between large and small scales (e.g., Elmegreen 2006). Furthermore, Zinnecker (1982) showed that an IMF slope $\gamma=2$ results simply from Bondi-Hoyle accretion $\dot{m} \propto m^{2}$ among the 
protostellar masses, with the mass function $n(m, t)$ evolving from some arbitrary initial distribution:

$$
\frac{d n}{d t}+\frac{d}{d m}(\dot{m} n)=0
$$

This yields a simple power-law IMF having $\gamma=2$ (see also Bonnell et al. 2007; Hsu et al. 2010). Thus $\gamma=2$ is a value that is intuitive, with simple and reasonable physical bases from both the parent ICMF equipartition arguments and buildup from simple accretion. So then why is the Salpeter slope of the IMF slightly steeper than $\gamma=2$ ?

Elmegreen (2009) summarizes the three approaches to generating the IMF: (1) fragmentation, in which the core mass function and IMF are a direct mapping from the parent cloud fragmentation; (2) accretion, in which the IMF is the product of protostar accretion processes not necessarily linked to how clouds fragment; and (3) interruption of (1) or (2), in which the form of the IMF is also determined by factors that preferentially limit fragmentation or accretion for high- or low-mass stars. If we adopt our argument above that, simplistically, the IMF should inherit $\gamma=2$ from the ICMF according to both (1) and (2), then (3) is a likely candidate to explain the deviation of the Salpeter slope: there is likely some process that limits the formation of high-mass stars in favor of low-mass ones.

In a fully hierarchical star formation scenario, the masses of the parent clouds and, hence, cluster masses are determined before the masses of the star-forming clumps, and in turn, the constituent stars. If the smallest clumps have masses on the order of stellar masses, then the formation of high-mass stars will necessarily be limited in such clumps, relative to the low-mass stars. In recent years, it has been proposed that the integrated galaxy IMF (IGIMF) has a steeper slope than the IMF because the most massive stars may not form in smallest clusters (Kroupa \& Weidner 2003; Weidner \& Kroupa 2005, 2006; see also Elmegreen 1999), causing the maximum stellar mass $m_{\mathrm{up}}$ to depend on the cluster mass. This would cause the IGIMF to favor lower-mass stars.

We propose that the difference between the Salpeter slope $\gamma=2.35$ and the ICMF slope $\beta=2$ is caused by a similar situation governing the distribution of stellar masses within individual clusters. If, following a fully hierarchical scenario, stellar subgroups are formed out of clumps with pre-determined masses, and if the clump mass distribution also generates a dependence of $m_{\text {up }}$ on clump mass $M$, then the resulting IMF for the entire cluster will be slightly steeper than the clump mass function. This scenario has essentially been proposed by Elmegreen (1997), who presented this as random sampling from a fractal mass distribution. Here, we present a parameterization that is much simpler than Elmegreen's model, and we show that the Salpeter slope simply results from the overlap between the stellar mass range and the clump mass range.

\section{RELATIVE MASS RANGES OF CLUMPS AND STARS}

We model the generation of stars in a cluster as the sum of all stars-forming from individual clumps in the parent molecular cloud. We assume that the hierarchical fragmentation of the cloud into clumps follows the same $\beta=2$ powerlaw distribution found for the ICMF, and further that the fragmentation of clumps into stars does the same, arguing that self-similar physical mechanisms govern all these fragmentation processes on smaller scales. This breaks down around the characteristic value of $m_{c} \sim 1 M_{\odot}$, suggested to be linked to the Jeans mass (e.g., McKee \& Ostriker 2007; Clarke 2009). Since we are interested only in the upper IMF, however, scale-free cloud fragmentation plausibly dominates in this regime. But we note that our model contrasts with those in which the IMF slope results from physics that causes fragmentation into a steeper core mass distribution (e.g., Padoan \& Nordlund 2002).

We construct Monte Carlo simulations drawing the clump mass function from the $\beta=2$ power law, and then the stellar mass function within each clump from the same distribution, within lower and upper stellar mass limits $m_{\mathrm{lo}}$ and $m_{\mathrm{up}}$. The entire cluster is then the aggregate of all the stars formed in all the clumps, and the IMF is the composite for this aggregate. Our default sampling algorithm allows rejection of stars that cause the clump mass limit to be exceeded, and continues sampling until the total stellar mass is within $m_{\mathrm{lo}}$ of the specified clump mass. This is similar to, but a bit more strongly sorted, than the "sorted sampling" algorithm of Weidner \& Kroupa (2006; see also Elmegreen 2006, Parker \& Goodwin 2007).

Figure 1 shows how the observed logarithmic IMF slope $\Gamma$, determined from fits weighted by the inverse of the poisson errors, depends on the mass ranges for the stars relative to the clumps. If the clump masses are all larger than the allowed stellar masses, then the stellar masses are unconstrained, and the parent $\beta=2$ distribution is reproduced, translating to $\Gamma=1$ on a logarithmic plot. This is apparent in Figure 1(a), having a fixed allowed stellar mass range of 1 to $100 M_{\odot}$, and clump upper-mass limit $M_{\text {up }}=10^{4} M_{\odot}$. Figure 1 (a) shows $\Gamma$ as a function of the lower clump limit $M_{\mathrm{lo}}$, and as $M_{\mathrm{lo}} \rightarrow m_{\mathrm{up}}$, $\Gamma \rightarrow 1$. But as allowed cloud masses decrease to values below $m_{\text {up }}$, the IMF slope steepens, since formation of the most massive stars is excluded. We see that as $M_{\mathrm{lo}} \rightarrow m_{\mathrm{lo}}$, the value approaches $\Gamma=1.4$, essentially the Salpeter value (black point). Figure 1(b) confirms that this effect is driven by the allowed clump lower-mass limit, rather than the upper limits on either the clumps or stars. This model allows both $M_{\text {up }}$ and $m_{\text {up }}$ to be essentially limitless, $10^{6} M_{\odot}$; the results are almost identical to Figure 1(a) in approaching the same Salpeter slope as $M_{\mathrm{lo}} \rightarrow m_{\mathrm{lo}}$. This steepening effect between $\beta=2$ and the Salpeter value can be seen in models by Elmegreen (1997) and Weidner \& Kroupa (2006). Indeed, Elmegreen's model allowed an effective equivalent $M_{\mathrm{lo}} \sim 1 M_{\odot}$, and Weidner \& Kroupa adopted an analogous $M_{\mathrm{lo}}=5 M_{\odot}$; both of these clump lowermass limits are well within the regime where the IMF steepening is expected, according to our simulations.

IMF slopes steeper than the Salpeter value can be obtained if both $M_{\text {lo }} \ll m_{\text {up }}$ and $M_{\text {up }}$ approaches stellar values. Figure 1(c) assumes $M_{\mathrm{lo}}=m_{\mathrm{lo}}$ and shows $\Gamma$ as a function of $M_{\mathrm{up}}$. For $M_{\text {up }} \gg m_{\text {up }}, \Gamma \sim 1.4$, as found before for the assumed $M_{\mathrm{lo}}=m_{\mathrm{lo}}$. As the clump upper-mass limit is decreased, $\Gamma$ steepens, reaching $\Gamma=1.9$ for $M_{\text {up }}=m_{\text {up }}=100 M_{\odot}$. The results are essentially identical even if $m_{\mathrm{up}}$ is limitless, although the maximum stellar mass will be limited by $M_{\text {up }}$ at low $M$. Weidner \& Kroupa (2006) also show that the degree to which the aggregate IMF steepens depends slightly on the sampling algorithm used. A "sorted sampling" algorithm, as we use, allows continued sampling of lower-mass stars after the highest-mass stars can no longer fit within the allotted mass, and naturally induces slightly steeper slopes than algorithms that stop sampling as soon as any drawn star causes the clump mass limit to be reached. Figure 1(d) is the same as panel (c), but with unsorted sampling, confirming that the sorting scheme is not a strong effect. 

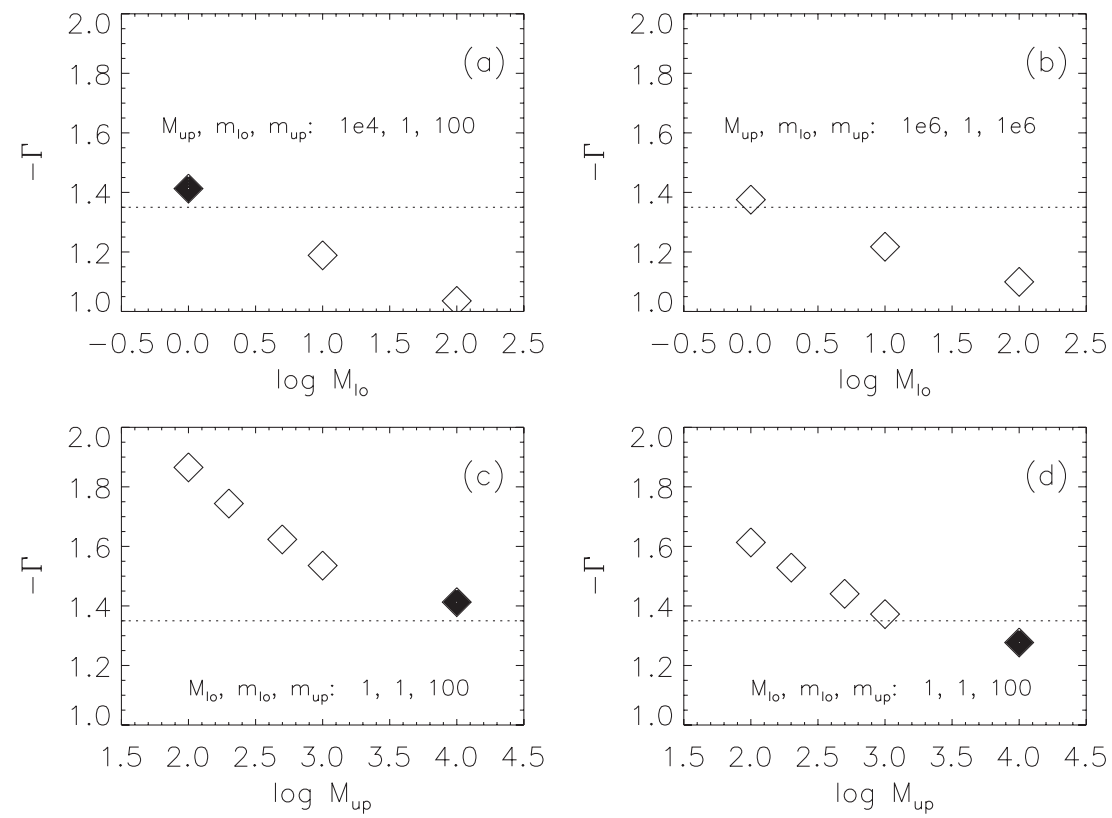

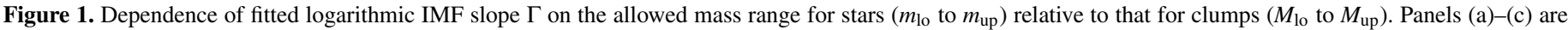

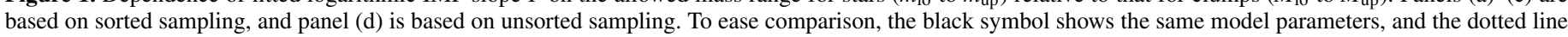
shows the Salpeter slope value.

Thus, we see that the slope steepening beyond the input $\beta=2$ value is driven by allowing clump masses $\ll m_{\text {up. In }}$ these lowest-mass clumps, the highest-mass stars cannot form, thus causing the aggregate cluster to slightly favor lower-mass stars beyond the conventional IMF. From a physical standpoint, this simply means that clumps too small to produce the highestmass stars can still produce low-mass stars. Furthermore, the Salpeter IMF results when $M_{\mathrm{lo}} \sim m_{\mathrm{lo}}$ while randomly sampling both stars and parent clumps from the same $\beta=2$ powerlaw distribution. Thus ironically, the critical parameter for steepening the IMF and the IGIMF is not $M_{\mathrm{up}}$, but rather $M_{\mathrm{lo}}$.

Elmegreen $(1997,1999)$ developed a model for IMF generation based on random sampling of stellar masses from hierarchical fractal structure. Our model distills the effects seen in Elmegreen's model to the most simplististic level, and it more directly captures the dominant effect seen in that work, revealing $M_{\mathrm{lo}}$ as the critical parameter.

\section{DISCUSSION}

The essential condition that drives the steepening of the IMF from $\gamma=2$ is that $M_{\mathrm{lo}} \ll m_{\mathrm{up}}$. This need not be interpreted literally, but the smallest clumps must be incapable of producing the highest-mass stars; our model is independent of star formation efficiency, provided that it is essentially constant. To attain the Salpeter value, in particular, requires the equivalent of $M_{\mathrm{lo}} \sim m_{\mathrm{lo}}$. Because the full IMF flattens strongly near a characteristic value $m_{c} \sim 1 M_{\odot}$, this limits the power-law form of the high-mass IMF to apply only above this characteristic mass, thus $m_{\text {lo }} \sim m_{c}$, recalling that here we consider only the upper, Salpeter IMF. Furthermore, because stellar objects continue to form with masses 1-2 orders of magnitude lower, clump fragmentation clearly continues to masses below $m_{c}$ as well, and so we know that the relevant lowest-mass clumps having $m_{\text {lo }} \sim m_{c}$ do form stars. Thus, for the power-law upper IMF, $M_{\mathrm{lo}} \leqslant m_{\mathrm{lo}}$, which is exactly the condition needed to induce steepening of the aggregate cluster IMF to the Salpeter value in the power-law regime above $m_{c}$. In other words, $m_{c}$ acts as an effective lower cutoff to the IMF power-law distribution for both clump and stellar masses, rendering $M_{\mathrm{lo}}=m_{\mathrm{lo}}$. Indeed, $\mathrm{C}^{18} \mathrm{O}$ observations of M17-SW show that molecular clump masses follow a power law of $\Gamma=-0.72 \pm 0.15$ down to at least $1 M_{\odot}$ (Stutzki \& Güsten 1990), while the observed IMF in the closely associated region M17-SWex shows $\Gamma=1.3 \pm 0.2$ (Povich \& Whitney 2010).

If clumps fragment according to a $\beta=2$ power law, then the range of observed IMF slopes should generally not fall below this value. Similarly, steeper IMF slopes reach $\Gamma \sim 2$ for $M_{\text {up }}=m_{\text {up }}=100 M_{\odot}$, when $M_{\text {lo }} \sim m_{\mathrm{lo}}$. A number of authors have shown compilations of measured cluster slopes, and $1 \leqslant \Gamma \leqslant 2$ does appear to be a fairly well-defined range of allowed slopes for the high-mass IMF (e.g., Elmegreen 1999, Figure 1; Bastian et al. 2010, Figure 2): what appears to be a large scatter about $\Gamma=1.35$ (Elmegreen 1999) can also be interpreted as variation within the range allowed by our model.

The basic premise of our model is that clumps fragment from clouds according to the $\beta=2$ power law and in particular that stars formed within each individual clump are still formed with a $\gamma=2$ power law. Thus, if newly formed stars can be identified with their natal sub-groups in the youngest star-forming regions, the IMF should appear to be closer to $\Gamma=1$ in such groups. It would be interesting to evaluate the IMF slopes for subgroups within massive star-forming regions, and to compare these to the aggregate cluster IMF. Also, observations focusing on specific subregions within extremely young clusters might be more likely to obtain IMF slopes closer to $\Gamma=1$, while more complete measurements for entire clusters might obtain the Salpeter value or higher. These are difficult tests, since dynamical evolution will quickly mix stars born from different clumps.

It is often suggested (e.g., Elmegreen 2004, 2009) that the IMF is slightly flatter in starbursts and rich, young, super star clusters. In our model, recovering an IMF slope of $\gamma=2$ occurs naturally if the clump mass $M_{\mathrm{lo}} \geqslant m_{\mathrm{up}}$. The Arches cluster near the Galactic Center is the best-known example of a flat IMF, with the most recent measurements of the high-mass IMF of 
$\Gamma=0.91 \pm 0.08$ (Kim et al. 2006) and $\Gamma=1.1 \pm 0.2$ (Espinoza et al. 2009); these values are consistent with our model. Regions of extreme star formation are expected to have higher thermal Jeans masses owing to radiative feedback from massive stars (e.g., Larson 2005; Murray 2009) therefore raising the minimum clump mass $M_{\mathrm{lo}}$. Our slope flattening would only occur if the clump mass range is increased without a commensurate increase in the allowed stellar mass range; and indeed, $m_{\text {up }}$ appears to be remarkably constant and independent of star formation environment (e.g., Oey \& Clarke 2005; Weidner \& Kroupa 2004).

If the smallest clusters form out of single clumps, with no further fragmentation, then the cluster IMF is the same as the clump IMF and there is no steepening of the aggregate slope. Hence, such single-clump clusters should show the parent $\gamma=2$ stellar IMF. Further, if the smallest clusters do approach limiting masses that are on the order of the largest clump masses, this should cause an analogous steepening of the cluster mass function under these circumstances. As described above, the ICMF generally has a slope $\beta=2$, but it would be interesting to evaluate any trends. We also note that our model does not necessarily imply an IGIMF steepening itself: smaller clusters can still fragment into clumps yielding the full stellar mass range for all, including the smallest clumps. So if the smallest clusters are still capable of forming stars up to masses $m_{\text {up }}$, as suggested by the results of Lamb et al. (2010), then no IGIMF steepening will occur. Thus again, the lower clump mass range relative to the upper stellar mass range determines the steepening for both the cluster stellar IMF and the IGIMF. We do however suggest that the IGIMF effect seen in low-density environments (e.g., Lee et al. 2009; Hoversten \& Glazebrook 2008) is real and occurs because the smallest clusters have masses below $m_{u p}$.

We stress that our model is a simplistic parameterization of hierarchical star formation, but it may capture essential elements that govern the generation of the Salpeter tail of the IMF. In particular, our model is based on a scenario that is fully hierarchical, with clouds fragmenting into clumps and clumps into stars, with each fragmentation process based on a $\beta=\gamma=2$ power-law mass distribution. "Fragmentation" need not be literal, but simply means that the ultimate mass apportionment from the parent to descendant units follows this power-law distribution, whether by competitive accretion or by well-defined fragmentation. The steepening of the IMF to the Salpeter value is then induced by the inability of the lowestmass clumps to form the highest-mass stars. Maschberger et al. (2010) have carried out a star formation simulation of a large volume, which follows the hierarchical fragmentation of ISM into clusters and down to core-like sink particles. Their results are consistent with our scenario, showing that clusters are mergers of products from multiple clumps, and that the aggregate IMF in such mergers is slightly steeper than in the individual merging units.

\section{CONCLUSION}

We propose that the empirical, logarithmic $\Gamma=1.35$ Salpeter power-law slope of the IMF for high-mass stars originates from a universal, linear $\gamma=2 \mathrm{IMF}$ slope for stellar sub-groups formed from clumps within individual clusters. Our Monte Carlo simulations show that $\Gamma=1.4$ is the limiting value of the IMF slope when the lower-mass limit for both clumps and stars is equal, $M_{\mathrm{lo}}=m_{\mathrm{lo}}$; this condition holds for the high-mass tail of the observed IMF, since it turns over below the characteristic mass $m_{c} \sim 1 M_{\odot}$, effectively setting the lower-mass limit to both $m_{\mathrm{lo}}$ and $M_{\mathrm{lo}}$. The steepening to the Salpeter value occurs because the highest-mass stars cannot form in the lowest-mass clumps. Thus, a critical factor to examine in star formation theory is the effective mass range for clumps relative to the output stellar masses. Our model is analogous to the mechanism for steepening the IGIMF proposed by Kroupa \& Weidner (2003), but a universal Salpeter slope does not necessarily imply that the IGIMF should steepen to even higher values, since the smallest clusters still may be capable of forming the highest-mass stars. However, we stress that the same principles involving the relative mass ranges of the parent and descendant units also apply to the IGIMF and may be responsible for the apparent steepening in regimes dominated by the smallest-scale star formation (e.g., Lee et al. 2009).

Our model is based on a fully hierarchical scenario in which the physics of fragmentation from large scales to individual stars takes place in a self-similar manner, all with a -2 power-law mass distribution. Thus, this does not require literal fragmentation, but simply that the final mass distribution among both the parent and descendant units is distributed accordingly. With this simple condition, we show that the aggregate highmass IMF slope is limited between values of $\Gamma=1$ and 2 , which is consistent with observations. Flatter values near $\Gamma=1$ occur when $M_{\mathrm{lo}}>m_{\mathrm{up}}$, which may explain the suggested IMF flattening for regions of extremely intense, massive star formation. While our model is only a rough parameterization of the star formation process, we suggest that it may capture the fundamental effects that generate the Salpeter slope of IMF for high-mass stars.

Thanks to Cathie Clarke, Joel Lamb, and Fred Adams for useful discussions and comments, and to the referee, Michael Reid. I gratefully acknowledge the Institute of Astronomy, Cambridge for visitor support, and funding by NSF grant AST0907758.

\section{REFERENCES}

Adams, F. C. 2003, Publ. Obs. Astron. Beogr., 75, 9

Bastian, N., Covey, K. R., \& Meyer, M. R. 2010, ARA\&A, 48, 339

Bonnell, I. A., Bate, M. R., \& Vine, S. G. 2003, MNRAS, 343, 413

Bonnell, I. A., Larson, R. B., \& Zinnecker, H. 2007, in Protostars and Planets V, ed. B. Reipurth, D. Jewitt, \& K. Keil (Tucson, AZ: Univ. Arizona Press), 149

Chabrier, G. 2003, PASP, 115, 763

Clarke, C. J. 2009, Ap\&SS, 324, 121

de Grijs, R., Andres, P., Bastian, N., et al. 2003, MNRAS, 343, 1285

Elmegreen, B. G. 1997, ApJ, 486, 944

Elmegreen, B. G. 1999, ApJ, 515, 323

Elmegreen, B. G. 2004, MNRAS, 354, 367

Elmegreen, B. G. 2006, ApJ, 648, 572

Elmegreen, B. G. 2009, in The Evolving ISM in the Milky Way and Nearby Galaxies, ed. K. Sheth, A. Noriega-Crespo, J. Ingalls, \& R. Paladini, arXiv:0803.3154

Elmegreen, B. G., \& Efremov, Y. N. 1997, ApJ, 480, 235

Espinoza, P., Selman, F. J., \& Melnick, J. 2009, A\&A, 501, 563

Hoversten, E. A., \& Glazebrook, K. 2008, ApJ, 675, 163

Hsu, W.-H., Hartmann, L. W., Heitsch, F., \& Gómez, G. C. 2010, ApJ, 721, 1531

Kennicutt, R. C., Edgar, B. K., \& Hodge, P. W. 1989, ApJ, 337, 761

Kim, S. S., Figer, D. F., Kudritzki, R. P., \& Najarro, F. 2006, ApJ, 653, L113

Kroupa, P. 2001, MNRAS, 322, 231

Kroupa, P., \& Weidner, C. 2003, ApJ, 598, 1076

Krumholz, M. R., Cunningham, A. J., Klein, R. I., \& McKee, C. F. 2010, ApJ, 713,1120

Lada, C. J. 2009, in Astrophysics in the Next Decade, ed. H. A. Thronson, M. Stiavelli, \& A. Tielens (Amsterdam: Springer), 147

Lada, C. J., \& Lada, E. A. 2003, ARA\&A, 41, 57

Lamb, J. B., Oey, M. S., Werk, J. K., \& Ingleby, L. D. 2010, ApJ, 725, 1886

Larson, R. B. 2005, MNRAS, 359, 211

Lee, J. C., Gil de Paz, A., Tremonti, C., et al. 2009, ApJ, 706, 599 
Maschberger, T., Clarke, C. J., Bonnell, I. A., \& Kroupa, P. 2010, MNRAS, 404, 1061

McKee, C. F., \& Ostriker, E. C. 2007, ARA\&A, 45, 565

Meurer, G. R., Heckman, T. M., Leitherer, C., et al. 1995, AJ, 110, 2665

Murray, N. 2009, ApJ, 691, 946

Oey, M. S., \& Clarke, C. J. 1998, AJ, 115, 1543

Oey, M. S., \& Clarke, C. J. 2005, ApJ, 620, L43

Oey, M. S., King, N. L., \& Parker, J. W. 2004, AJ, 127, 1632

Padoan, P., \& Nordlund, Å. 2002, ApJ, 576, 870
Parker, R. J., \& Goodwin, S. P. 2007, MNRAS, 380, 1271

Povich, M. S., \& Whitney, B. A. 2010, ApJ, 714, L285

Salpeter, E. E. 1955, ApJ, 121, 161

Stutzki, J., \& Güsten, R. 1990, ApJ, 356, 513

Weidner, C., \& Kroupa, P. 2004, MNRAS, 348, 187

Weidner, C., \& Kroupa, P. 2005, ApJ, 625, 754

Weidner, C., \& Kroupa, P. 2006, MNRAS, 365, 1333

Zhang, Q., \& Fall, S. M. 1999, ApJ, 527, L81

Zinnecker, H. 1982, Ann. NY Acad. Sci., 395, 226 